\title{
Matrix elements of $\mathrm{SU}(6)$ generators for baryons with arbitrary $N_{c}$ quarks in mixed symmetric states $\left[N_{c}-1,1\right]$
}

\author{
N. Matagne ${ }^{a *}$ and Fl. Stancu ${ }^{b \dagger}$ \\ a Institut für Theoretische Physik, Universität Gießen, D-35392 Gießen, Germany \\ ${ }^{b}$ University of Liège, Institute of Physics B5, \\ Sart Tilman, B-4000 Liège 1, Belgium
}

(Dated: October 24, 2018)

\begin{abstract}
We derive general analytic formulae for the matrix elements of the $\mathrm{SU}(6)$ generators for mixed symmetric $\left[N_{c}-1,1\right]$ spin-flavor states with an arbitrary number $N_{c}$ of quarks. They are relevant for baryon spectroscopy in the $1 / N_{c}$ expansion method applied to light baryons and can be used to study excited states. In this way previous work on non-strange baryons can be extended to both non-strange and strange baryons.
\end{abstract}

\footnotetext{
* e-mail address: Nicolas.Matagne@theo.physik.uni-giessen.de

$\dagger$ e-mail address: fstancu@ulg.ac.be
} 


\section{INTRODUCTION}

In the $1 / N_{c}$ expansion method [1, 2] the ground state baryons have an approximate $\mathrm{SU}\left(2 N_{f}\right)$ symmetry when the number of colors $N_{c}$ is large but finite. This stems from the property that when $N_{c} \rightarrow \infty$ the $\mathrm{SU}\left(2 N_{f}\right)$ is an exact contracted symmetry [3, 4] and in that limit the baryons are degenerate. At large but finite $N_{c}$ the mass splitting starts at order $1 / N_{c}$ for the ground or excited symmetric states and at order $N_{c}^{0}$ for mixed symmetric states.

Here we consider light baryons with $N_{f}=3$, thus we deal with $\mathrm{SU}(6)$ symmetry. In this case the building blocks of the mass operator are the generators of SU(6). For the excited states the symmetry is extended to $\mathrm{SU}(6) \times \mathrm{SO}(3)$. Therefore the generators of $\mathrm{SO}(3)$ also appear in the mass formula.

The study of excited states of symmetric orbital symmetry is straightforward. However the study of excited states of mixed orbital symmetry, or equivalently mixed spin-flavor symmetry, presented so far some difficulty related to the fact the matrix elements of SU(6) generators between mixed symmetric $\left[N_{c}-1,1\right]$ spin-flavor states were unknown. Accordingly, a method based on the separation of a system of $N_{c}$ quarks into a symmetric "core" of $N_{c}-1$ quarks and an excited quark was proposed [5] and applied to the [70, $1^{-}$] and $\left[\mathbf{7 0}, \ell^{+}\right](\ell=0,2)$ multiplets, for $N_{f}=2[\underline{5}, \underline{6}]$ and $N_{f}=3[7$, 8]. The orbital-spin-flavor wave function describing such a decoupled system is not totally symmetric, as it should be. Its approximate form is explained at large in Ref. [9]. In addition, to match the decoupling,

in Ref. [5] each generator of $\mathrm{SU}\left(2 N_{f}\right)$ was written as a sum of two terms, one acting on the core and the other on the excited quark. As a consequence, the number of linearly independent operators appearing in the mass formula increases and the number of coefficients to be determined generally becomes larger or nearly as large as the number of the experimental data available. For example, for the $\left[\mathbf{7 0}, 1^{-}\right]$multiplet with $N_{f}=3$ one has at least 15 linearly independent operators up to order $1 / N_{c}$ included [5] and 7 known masses. Then one must select the most dominant operators, which is a very difficult task, not free from ambiguities $[7]$.

In Ref. [10] we have proposed a new approach where the separation of the system into a symmetric core of $N_{c}-1$ quarks and an excited quark can be avoided. The approach was restricted to $N_{f}=2$. In this way the number of linearly independent operators was 
substantially reduced. In addition the method has important physical consequences. We have shown for example that the term containing the isospin-isospin interaction in the mass formula of $\Delta$, neglected in all previous applications, becomes as dominant as the pure spinspin term in $N$. To obtain such results the matrix elements of the generators of $\mathrm{SU}(4)$ were needed. General analytic expression were available from nuclear physics studies [11]. It was easy enough to adjust them to a system of $N_{c}$ quarks.

Let us explain the previous situation in detail. In the scheme based on the separation of the system into a symmetric core of $N_{c}-1$ quarks and an excited quark the $\mathrm{SU}(2)$-isospin Casimir operator was written as $T^{2}=T_{c}^{2}+2 t \cdot T_{c}+3 / 4$, where the lower index $c$ refers to the core, and decomposed into three independent pieces, corresponding to the terms in the above decomposition. In $\mathrm{SU}(4) T_{c}^{2}$ and $S_{c}^{2}$ have identical matrix elements because the spin and isospin states of a symmetric core are identical, so that $T_{c}^{2}$ can be neglected. But $t \cdot T_{c}$ has different matrix elements from $s \cdot S_{c}$ as one can clearly see from Table II of Ref. [5]. Then in the decoupling scheme the isospin can be introduced only through $t \cdot T_{c}$. In Ref. [9] Table VI we have shown that the introduction of the operators $\frac{1}{N_{c}} t \cdot T_{c}$ together with $\frac{1}{N_{c}} S_{c}^{2}$ and $\frac{1}{N_{c}} s \cdot S_{c}$ separately deteriorates the fit. This may explain why $\frac{1}{N_{c}} t \cdot T_{c}$ has been avoided in previous numerical fits both in $\mathrm{SU}(4)$ [5] and in $\mathrm{SU}(6)$ [7]. We avoided it as well [6] in line with our predecessors.

To extend the application of the method of Ref. [10] from non-strange to both nonstrange and strange baryons one needs to know the matrix elements of the generators of $\mathrm{SU}(6)$. In this work we derive these matrix elements.

We recall that the group $\mathrm{SU}(6)$ has 35 generators $S^{i}, T^{a}, G^{i a}$ with $i=1,2,3$ and $a=$ $1,2, \ldots, 8$ where $S^{i}$ are the generators of the spin subgroup $\mathrm{SU}(2)$ and $T^{a}$ the generators of the flavor subgroup $\mathrm{SU}(3)$. The group algebra is

$$
\begin{gathered}
{\left[S^{i}, S^{j}\right]=i \varepsilon^{i j k} S^{k}, \quad\left[T^{a}, T^{b}\right]=i f^{a b c} T^{c},} \\
{\left[S^{i}, G^{j a}\right]=i \varepsilon^{i j k} G^{k a}, \quad\left[T^{a}, G^{j b}\right]=i f^{a b c} G^{i c},} \\
{\left[G^{i a}, G^{j b}\right]=\frac{i}{4} \delta^{i j} f^{a b c} T^{c}+\frac{i}{2} \varepsilon^{i j k}\left(\frac{1}{3} \delta^{a b} S^{k}+d^{a b c} G^{k c}\right),}
\end{gathered}
$$

by which the normalization of the generators is fixed.

We redefine the generators forming the algebra (11) as

$$
E^{i}=\frac{S^{i}}{\sqrt{3}} ; \quad E^{a}=\frac{T^{a}}{\sqrt{2}} ; \quad E^{i a}=\sqrt{2} G^{i a} .
$$


Note that the generic name for every generator will also be $E^{i a}$ [11]. Specifications will be made whenever necessary. Here we search for the matrix elements of $S^{i}, T^{a}$ and $G^{i a}$ between $\mathrm{SU}(6)$ states of symmetry $\left[N_{c}-1,1\right]$. As we shall see below, the matrix elements of $S^{i}$ and $T^{a}$ are straightforward. The remaining problem is to derive the matrix elements of $G^{i a}$.

The SU(6) generators are the components of an irreducible tensor operator which transform according to the adjoint representation $\left[21^{4}\right]$, equivalent to 35 , in dimensional notation. There are several ways to calculate the matrix elements of the $\mathrm{SU}(6)$ generators. In the standard group theory the matrix elements of any irreducible tensor can be expressed in terms of a generalized Wigner-Eckart theorem which is a factorization theorem, involving the product between a reduced matrix element and a Clebsch-Gordan (CG) coefficient. The CG coefficient of $\mathrm{SU}(6)$ factorizes into $\mathrm{CG}$ coefficients of $\mathrm{SU}(2), \mathrm{SU}(3)$ and an isoscalar factor of $\mathrm{SU}(6)$, see Eq. (13). The latter is the quantity we derive here.

In the 60'ties the literature provided a few examples of isoscalar factors needed in particle physics, thus for $N_{c}=3$. Cook and Murtanza [12] considered the full CG series of the direct products $35 \times \mathbf{3 5}, \mathbf{5 6} \times \mathbf{3 5}$ and $\mathbf{5 6} \times \overline{\mathbf{5 6}}$. Carter, Coyne and Meshkov [13] derived the isoscalar factors for $\mathbf{5 6} \times \mathbf{3 5} \rightarrow \mathbf{5 6}$ independently from Schülke [14] who also calculated the isoscalar factors for $3 \mathbf{5} \times \mathbf{3 5} \rightarrow \mathbf{3 5}$ like Cook and Murtanza. Moreover Carter and Coyne [15] derived the isoscalar factors of the whole CG series $35 \times \mathbf{7 0}=\mathbf{2 0}+\mathbf{5 6}+2 \times \mathbf{7 0}+\mathbf{5 4 0}+\mathbf{5 6 0}+\mathbf{1 1 3 4}$. In Ref. [16] we have obtained analytic formulae for isoscalar factors of arbitrary $N_{c}$ which for $N_{c}=3$ correspond to $56 \times \mathbf{3 5} \rightarrow \mathbf{5 6}$. Up to a phase we have found full agreement with Refs. [12, 13, 14]. In the present case, by setting $N_{c}=3$ in our formulae, we could, in principle, compare the results with either column $70_{\text {I }}$ or column $70_{\text {II }}$ of Ref. [15], for $\mathbf{3 5} \times \mathbf{7 0} \rightarrow \mathbf{7 0}$. In fact, following our definition, one does not need to compute the isoscalar factors of both $70_{\text {I }}$ and $70_{\mathrm{II}}$ to derive the matrix elements of the generators. However a multiplicity 2 problem can appear in the direct product of two $\mathrm{SU}(3)$ irreducible representations (see Eq. (18) below). For $N_{c}=3$, this is the case for the product $(8 \times 8) \rightarrow 8$. Here we shall use the label $\rho$ [16] to distinguish between the two representations when the multiplicity is 2. In Ref. [15], the authors follow the notation of [17] by using the label $S$ for the symmetric product and $A$ for antisymmetric product corresponding respectively to $\rho=2$ and $\rho=1$ in our notation. For consistency with previous work [16], we follow the definition of Ref. [11], (see Eq. (16) below), which simplifies the problem, in the sense that we obtain vanishing $\mathrm{SU}(6)$ isoscalar factors for the products $\left(8^{4,2} \times 8^{1}\right)_{\mathrm{S}}$ while Carter and Coyne obtain non-vanishing values for 
the isoscalar factors for these products. Thus the comparison is impossible. Such ambiguities are typical for all groups, including the permutation group, whenever the multiplicity in the CG series is larger than one [18]. As mentioned above, for unitary groups, following GellMann, it is customary to introduce the symmetric $S$ or $D$ coupling and antisymmetric $A$ or $F$ coupling. The choice is based on convenience anyhow [19].

The paper is organized as follows. In the next section we introduce the $\mathrm{SU}(6)$ basis states. In Sec. III we remind the generalized Wigner-Eckart theorem which allows a factorization of the matrix elements into Clebsch-Gordan coefficients and some specific isoscalar factors. In Sec. IV we derive the unknown isoscalar factors. In Sec. V we discuss possible physical applications to the mass spectrum and in the last section we summarize our results.

\section{THE WAVE FUNCTION}

We deal with a system of $N_{c}$ quarks having one unit of orbital excitation. Therefore the orbital $(O)$ wave function must have a mixed symmetry $\left[N_{c}-1,1\right]$, which describes the lowest excitations in a baryon. The fact that this is the lowest excitation with $L=1$ is well known in group theory and has been extensively applied to nuclear shell model, see e.g. [20] or [21]. Moreover the $N_{c}-1$ independent basis states of the $\left[N_{c}-1,1\right]$ irrep written in the Young-Yamanouchi basis, see below, is equivalent to a basis written in terms of $N_{c}-1$ internal Jacobi coordinates, thus the center of mass motion is automatically removed. An example for four quarks can be found in Ref. [22].

The colour wave function being antisymmetric, the orbital-spin-flavor wave part must be symmetric. Then the spin-flavor $(F S)$ part must have the same symmetry as the orbital part in order to obtain a totally symmetric state in the orbital-spin-flavor space. The general form of such a wave function is [23]

$$
\left|\left[N_{c}\right]\right\rangle=\frac{1}{\sqrt{d_{\left[N_{c}-1,1\right]}}} \sum_{Y}\left|\left[N_{c}-1,1\right] Y\right\rangle_{O}\left|\left[N_{c}-1,1\right] Y\right\rangle_{F S}
$$

where $d_{\left[N_{c}-1,1\right]}=N_{c}-1$ is the dimension of the representation $\left[N_{c}-1,1\right]$ of the permutation group $S_{N_{c}}$ and $Y$ is a symbol for a Young tableau (Yamanouchi symbol). The sum is performed over all possible standard Young tableaux. In each term the first basis vector represents the orbital space and the second the spin-flavor space. In this sum there is only one $Y$ (the normal Young tableau) where the last particle is in the second row and $N_{c}-2$ 
terms where the last particle is in the first row. The explicit form of the orbital part is not needed.

More precisely, we write $Y=(p q y)$ where $p$ is the row of the $N_{c}$-th particle, $q$ the row of the $\left(N_{c}-1\right)$-th particle and $y$ is the Young tableau of the remaining particles. Let us denote by $p, p^{\prime}$ and $p^{\prime \prime}$ the position of the last particle in the spin-flavor, spin and flavor Young tableaux respectively. They are indicated by crosses in the example given by Eqs. (8)-(11) below. Similarly for the $\left(N_{c}-1\right)$-th particle we have $q, q^{\prime}$ and $q^{\prime \prime}$ and for the rest $y, y^{\prime}$ and $y^{\prime \prime}$. We need now to decompose the spin-flavor wave function into its spin and flavor parts separately. For this purpose we use the Clebsch-Gordan (CG) coefficients of $S_{N_{c}}$, denoted by $S\left(\left[f^{\prime}\right] p^{\prime} q^{\prime} y^{\prime}\left[f^{\prime \prime}\right] p^{\prime \prime} q^{\prime \prime} y^{\prime \prime} \mid[f] p q y\right)$ and their factorization property [23]. Denoting by $K\left(\left[f^{\prime}\right] p^{\prime}\left[f^{\prime \prime}\right] p^{\prime \prime} \mid[f] p\right)$ the isoscalar factors of $S_{N_{c}}$ we have $[9]$

$$
S\left(\left[f^{\prime}\right] p^{\prime} q^{\prime} y^{\prime}\left[f^{\prime \prime}\right] p^{\prime \prime} q^{\prime \prime} y^{\prime \prime} \mid[f] p q y\right)=K\left(\left[f^{\prime}\right] p^{\prime}\left[f^{\prime \prime}\right] p^{\prime \prime} \mid[f] p\right) S\left(\left[f_{p^{\prime}}^{\prime}\right] q^{\prime} y^{\prime}\left[f_{p^{\prime \prime}}^{\prime \prime}\right] q^{\prime \prime} y^{\prime \prime} \mid\left[f_{p}\right] q y\right),
$$

where the second factor in the right-hand side is a CG coefficient of $S_{N_{c}-1}$ containing the partitions $\left[f_{p^{\prime}}^{\prime}\right],\left[f_{p^{\prime \prime}}^{\prime \prime}\right]$ and $\left[f_{p}\right]$ obtained after the removal of the $N_{c}$-th quark.

Using the above property we can write the spin-flavor part of the wave function as

$$
\left|\left[N_{c}-1,1\right] p ;(\lambda \mu) Y I I_{3} ; S S_{3}\right\rangle=\sum_{p^{\prime} p^{\prime \prime}} K\left(\left[f^{\prime}\right] p^{\prime}\left[f^{\prime \prime}\right] p^{\prime \prime} \mid\left[N_{c}-1,1\right] p\right)\left|S S_{3} ; p^{\prime}\right\rangle\left|(\lambda \mu) Y I I_{3} ; p^{\prime \prime}\right\rangle
$$

where the spin part is

$$
\left|S S_{3} ; p^{\prime}\right\rangle=\sum_{m_{1}, m_{2}}\left(\begin{array}{cc|c}
S_{c} & \frac{1}{2} & S \\
m_{1} & m_{2} & S_{3}
\end{array}\right)\left|S_{c} m_{1}\right\rangle\left|1 / 2 m_{2}\right\rangle
$$

with $S_{c}=S-1 / 2$ if $p^{\prime}=1$ and $S_{c}=S+1 / 2$ if $p^{\prime}=2$ and the flavor part is

$$
\begin{aligned}
& \left|(\lambda \mu) Y I I_{3}, p^{\prime \prime}\right\rangle= \\
& \sum_{\substack{Y_{c}, I_{c}, I_{c_{3}}, y i i_{3}}}\left(\begin{array}{cc||c}
\left(\lambda_{c} \mu_{c}\right) & (10) \\
Y_{c} I_{c} & y i & (\lambda \mu) \\
Y I
\end{array}\right)\left(\begin{array}{cc|c}
I_{c} & i & I \\
I_{c_{3}} & i_{3} & I_{3}
\end{array}\right)\left|\left(\lambda_{c} \mu_{c}\right) Y_{c} I_{c} I_{c_{3}}\right\rangle\left|(10) y i i_{3}\right\rangle,
\end{aligned}
$$

with $\left(\lambda_{c}, \mu_{c}\right)=(\lambda-1, \mu)$ for $p^{\prime \prime}=1,\left(\lambda_{c}, \mu_{c}\right)=(\lambda+1, \mu-1)$ for $p^{\prime \prime}=2$ and $\left(\lambda_{c}, \mu_{c}\right)=(\lambda, \mu+1)$ for $p^{\prime \prime}=3$. Each $\mathrm{SU}(3)$ irreducible representation carries the label $(\lambda \mu)$.

Let us illustrate Eq. (5) in terms of Young tableaux by taking the $N_{c}=7$ and $p=2$. We have the following cases 

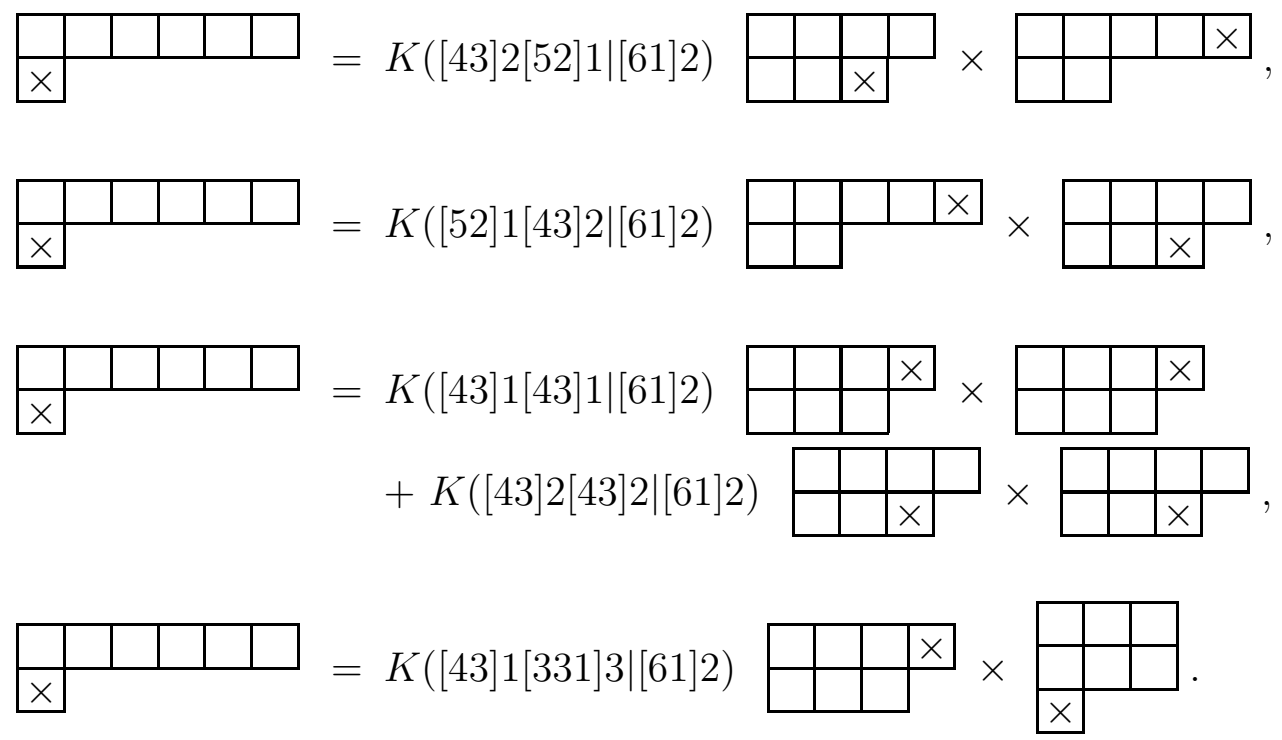

When $N_{c}=3$ the above spin-flavor states correspond to ${ }^{2} 10,{ }^{4} 8,{ }^{2} 8$ and ${ }^{2} 1$ multiplets. For the purpose of the present study we actually need only the case $p=2$. In this case the isoscalar factors $K\left(\left[f^{\prime}\right] p^{\prime}\left[f^{\prime \prime}\right] p^{\prime \prime} \mid\left[N_{c}-1,1\right] p\right)$ for arbitrary $N_{c}$ have the following algebraic form [6]

$$
\begin{aligned}
K\left(\left[\frac{N_{c}+1}{2}, \frac{N_{c}-1}{2}\right] 2\left[\frac{N_{c}+3}{2}, \frac{N_{c}-3}{2}\right] 1 \mid\left[N_{c}-1,1\right] 2\right) & =1 \\
K\left(\left[\frac{N_{c}+3}{2}, \frac{N_{c}-3}{2}\right] 1\left[\frac{N_{c}+1}{2}, \frac{N_{c}-1}{2}\right] 1 \mid\left[N_{c}-1,1\right] 2\right) & =1 \\
K\left(\left[\frac{N_{c}+1}{2}, \frac{N_{c}-1}{2}\right] 1\left[\frac{N_{c}+1}{2}, \frac{N_{c}-1}{2}\right] 1 \mid\left[N_{c}-1,1\right] 2\right) & =-\sqrt{\frac{3\left(N_{c}-1\right)}{4 N_{c}}}, \\
K\left(\left[\frac{N_{c}+1}{2}, \frac{N_{c}-1}{2}\right] 2\left[\frac{N_{c}+1}{2}, \frac{N_{c}-1}{2}\right] 2 \mid\left[N_{c}-1,1\right] 2\right) & =\sqrt{\frac{N_{c}+3}{4 N_{c}}} \\
K\left(\left[\frac{N_{c}+1}{2}, \frac{N_{c}-1}{2}\right] 1\left[\frac{N_{c}-1}{2}, \frac{N_{c}-1}{2}, 1\right] 3 \mid\left[N_{c}-1,1\right] 2\right) & =1 .
\end{aligned}
$$

For completeness we mention that the isoscalar factors for $p=1$ and non-strange states can be found in Ref. [9].

\section{MATRIX ELEMENTS OF SU(6) GENERATORS: THE GENERALIZED WIGNER-ECKART THEOREM}

For the spin $S^{i}$ and the flavor $T^{a}$ operators the matrix elements can be obtained from the Wigner-Eckart theorem in a similar manner as for symmetric $N_{c}$ states [16]. As already 
mentioned, the SU(6) generators are the components of an irreducible tensor operator which transform according to the adjoint representation $\left[21^{4}\right]$, equivalent to 35 , in dimensional notation. The matrix elements of any irreducible tensor can be expressed in terms of a generalized Wigner-Eckart theorem which is a factorization theorem, involving the product between a reduced matrix element and a Clebsch-Gordan (CG) coefficient. The case SU(4) $\supset \mathrm{SU}(2) \times \mathrm{SU}(2)$ has been worked out by Hecht and Pang [11] in a general form needed for applications to nuclear physics.

By using the generalized Wigner-Eckart theorem, in Ref. [16] we have derived explicit formulas for the matrix elements of $\mathrm{SU}(6)$ generators for symmetric states of $N_{c}$ quarks of partition $\left[N_{c}\right]$. Here we use a different procedure to obtain the matrix elements of $\mathrm{SU}(6)$ generators between mixed symmetric states $\left[N_{c}-1,1\right]$. When $N_{c}=3$ they correspond to the representation $[\mathbf{7 0}]$.

By analogy to SU(4) [11] one can write the matrix elements of every $\mathrm{SU}(6)$ generator $E^{i a}$ as

$$
\begin{aligned}
& \left\langle\left[N_{c}-1,1\right]\left(\lambda^{\prime} \mu^{\prime}\right) Y^{\prime} I^{\prime} I_{3}^{\prime} S^{\prime} S_{3}^{\prime}\left|E^{i a}\right|\left[N_{c}-1,1\right](\lambda \mu) Y I I_{3} S S_{3}\right\rangle= \\
& \quad \sqrt{C^{\left[N_{c}-1,1\right]}(\mathrm{SU}(6))}\left(\begin{array}{cc|c}
S & S^{i} & S^{\prime} \\
S_{3} & S_{3}^{i} & S_{3}^{\prime}
\end{array}\right)\left(\begin{array}{cc|c}
I & I^{a} & I^{\prime} \\
I_{3} & I_{3}^{a} & I_{3}^{\prime}
\end{array}\right) \\
& \quad \times \sum_{\rho=1,2}\left(\begin{array}{cc|c}
(\lambda \mu) & \left(\lambda^{a} \mu^{a}\right) \\
Y I & Y^{a} I^{a} & \left(\lambda^{\prime} \mu^{\prime}\right) \\
Y^{\prime} I^{\prime}
\end{array}\right)_{\rho}\left(\begin{array}{cc}
{\left[N_{c}-1,1\right]} & {\left[21^{4}\right]} \\
(\lambda \mu) S & \left(\lambda^{a} \mu^{a}\right) S^{i}
\end{array} \mid \begin{array}{c}
{\left[N_{c}-1,1\right]} \\
\left(\lambda^{\prime} \mu^{\prime}\right) S^{\prime}
\end{array}\right)_{\rho},
\end{aligned}
$$

where $C^{\left[N_{c}-1,1\right]}(\mathrm{SU}(6))=N_{c}\left(5 N_{c}+18\right) / 12$ is the $\mathrm{SU}(6)$ Casimir operator associated to the irreducible representation $\left[N_{c}-1,1\right]$, followed by Clebsch-Gordan coefficients of SU(2)-spin and $\mathrm{SU}(2)$-isospin. The sum over $\rho$ is over terms containing products of isoscalar factors of $\mathrm{SU}(3)$ and $\mathrm{SU}(6)$ respectively. In particular, $T^{a}$ is an $\mathrm{SU}(3)$ irreducible tensor operator of components $T_{Y^{a} I^{a}}^{(11)}$, i.e. a corresponds to $\left(\lambda^{a} \mu^{a}\right)$. It is a scalar in $\mathrm{SU}(2)$ so that the index $i$ from $E^{i a}$ is no more necessary. The generators $S^{i}$ form a rank 1 tensor in $\mathrm{SU}(2)$ which is a scalar in $\mathrm{SU}(3)$, so the index $i$ suffices. Although we use the same symbol for the operator $S^{i}$ and its quantum numbers we hope that no confusion is created. Thus, for the generators $S^{i}$ and $T^{a}$, which are elements of the $s u(2)$ and $s u(3)$ subalgebras of (1), the above expression simplifies considerably. In particular, as $S^{i}$ acts only on the spin part of the wave function, we apply the usual Wigner-Eckart theorem for $\mathrm{SU}(2)$ to get

$$
\left\langle\left[N_{c}-1,1\right]\left(\lambda^{\prime} \mu^{\prime}\right) Y^{\prime} I^{\prime} I_{3}^{\prime} ; S^{\prime} S_{3}^{\prime}\left|S^{i}\right|\left[N_{c}-1,1\right](\lambda \mu) Y I I_{3} ; S S_{3}\right\rangle=
$$




$$
\delta_{S S^{\prime}} \delta_{\lambda \lambda^{\prime}} \delta_{\mu \mu^{\prime}} \delta_{Y Y^{\prime}} \delta_{I I^{\prime}} \delta_{I_{3} I_{3}^{\prime}} \sqrt{C(\mathrm{SU}(2))}\left(\begin{array}{cc|c}
S & 1 & S^{\prime} \\
S_{3} & i & S_{3}^{\prime}
\end{array}\right)
$$

with $C(\mathrm{SU}(2))=S(S+1)$. Similarly, we use the Wigner-Eckart theorem for $T^{a}$ which is a generator of the subgroup $\mathrm{SU}(3)$

$$
\begin{aligned}
& \left\langle\left[N_{c}-1,1\right]\left(\lambda^{\prime} \mu^{\prime}\right) Y^{\prime} I^{\prime} I_{3}^{\prime} ; S^{\prime} S_{3}^{\prime}\left|T^{a}\right|\left[N_{c}-1,1\right](\lambda \mu) Y I I_{3} ; S S_{3}\right\rangle= \\
& \delta_{S S^{\prime}} \delta_{S_{3} S_{3}^{\prime}} \delta_{\lambda \lambda^{\prime}} \delta_{\mu \mu^{\prime}} \sum_{\rho=1,2}\left\langle\left(\lambda^{\prime} \mu^{\prime}\right)\left\|T^{(11)}\right\|(\lambda \mu)\right\rangle_{\rho}\left(\begin{array}{cc|c}
(\lambda \mu) & (11) & \left(\lambda^{\prime} \mu^{\prime}\right) \\
Y I I_{3} & Y^{a} I^{a} I_{3}^{a} & Y^{\prime} I^{\prime} I_{3}^{\prime}
\end{array}\right),
\end{aligned}
$$

where the reduced matrix element is defined as [24]

$$
\left\langle(\lambda \mu)\left\|T^{(11)}\right\|(\lambda \mu)\right\rangle_{\rho}=\left\{\begin{array}{cc}
\sqrt{C(\mathrm{SU}(3))} & \text { for } \rho=1 \\
0 & \text { for } \rho=2
\end{array},\right.
$$

in terms of the eigenvalue of the Casimir operator $C(\mathrm{SU}(3))=\frac{1}{3} g_{\lambda \mu}$ where

$$
g_{\lambda \mu}=\lambda^{2}+\mu^{2}+\lambda \mu+3 \lambda+3 \mu .
$$

Note that the presence of the index $\rho$ has the same origin as in Eq. (13), namely it reflects the multiplicity problem appearing in the direct product of $\mathrm{SU}(3)$ irreducible representations

$$
\begin{aligned}
& (\lambda \mu) \times(11)=(\lambda+1, \mu+1)+(\lambda+2, \mu-1)+(\lambda \mu)_{1}+(\lambda \mu)_{2} \\
& \quad+(\lambda-1, \mu+2)+(\lambda-2, \mu+1)+(\lambda+1, \mu-2)+(\lambda-1, \mu-1) .
\end{aligned}
$$

Each SU(3) CG coefficient factorizes into an SU(2)-isospin CG coefficient and an SU(3) isoscalar factor [17]

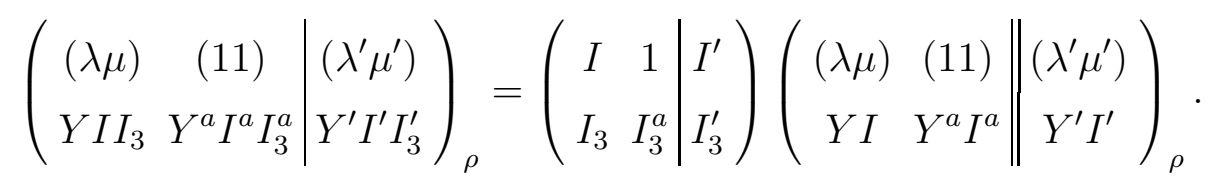

The analytic expression of the isoscalar factors can be found in Table 4 of Ref. [24].

Therefore the basic problem is to determine the matrix elements of $G^{i a}$. The procedure is described in the next section.

\section{SU(6) ISOSCALAR FACTORS}

\section{A. The general procedure}

A convenient way to derive the matrix elements of $G^{i a}$ is by decoupling the $N_{c}$-th quark from the system of $N_{c}$ quarks in a mixed symmetric state $\left[N_{c}-1,1\right]$. Let us denote by $G^{i a}$, 
$G_{c}^{i a}$ and $g^{i a}$ the generators of the total, of the $N_{c}-1$ system and of the decoupled quark. Then one has

$$
G^{i a}=G_{c}^{i a}+g^{i a}
$$

As mentioned before the last quark can be either in the row $p=1$ or in $p=2$. Now we use the important observation that the matrix elements of the generators are independent of the choice of $p$ as used, for example, in Appendix A of Ref. [9]. The explanation lies in Weyl's duality theorem according to which the basis vectors introduced in Sec. II form invariant subspaces both for the permutation group and the SU(6) group [23]. It is therefore useful to take $p=2$ because in that case the system of $N_{c}-1$ quarks is in a symmetric $\left[N_{c}-1\right]$ state for which the matrix elements are already known from Ref. [16] where it is enough to replace $N_{c}$ by $N_{c}-1$. The matrix elements of $g^{i a}$ are the trivial case of symmetric states with $N_{c}=1$. In a short notation we therefore have

$$
\left\langle G^{i a}\right\rangle=\left\langle G_{c}^{i a}\right\rangle_{p}+\left\langle g^{i a}\right\rangle_{p}
$$

irrespective of the value $p=1$ or 2 .

After lengthy calculations we obtain the following expression for the matrix elements in the right-hand side of (21)

$$
\begin{aligned}
& \left\langle\left[N_{c}-1,1\right] p ;\left(\lambda^{\prime} \mu^{\prime}\right) Y^{\prime} I^{\prime} I_{3}^{\prime} ; S^{\prime} m_{s}^{\prime}\left|G_{c}^{j a}\right|\left[N_{c}-1,1\right] p ;(\lambda \mu) Y I I_{3} ; S m_{s}\right\rangle= \\
& (-1)^{1 / 2-S} \sqrt{(2 S+1)\left(2 S_{c}^{\prime}+1\right)} \sqrt{\frac{C^{[f]}(S U(6))}{2}}\left(\begin{array}{cc|c}
S & 1 & S^{\prime} \\
m_{s} & j & m_{s}^{\prime}
\end{array}\right)\left(\begin{array}{cc|c}
I & I^{a} & I^{\prime} \\
I_{3} & I_{3}^{a} & I_{3}^{\prime}
\end{array}\right) \\
& \times \sum_{p^{\prime}, p^{\prime \prime}, q^{\prime}, q^{\prime \prime}}(-1)^{S_{c}^{\prime}}(-1)^{\lambda-\lambda_{c}+\lambda^{\prime}-\lambda_{c}^{\prime}}(-1)^{\mu-\mu_{c}+\mu^{\prime}-\mu_{c}^{\prime}} K\left(\left[f^{\prime}\right] p^{\prime}\left[f^{\prime \prime}\right] p^{\prime \prime} \mid\left[N_{c}-1,1\right] p\right) \\
& \times K\left(\left[f^{\prime}\right] q^{\prime}\left[f^{\prime \prime}\right] q^{\prime \prime} \mid\left[N_{c}-1,1\right] p\right)\left\{\begin{array}{ccc}
S & 1 & S^{\prime} \\
S_{c}^{\prime} & 1 / 2 & S_{c}
\end{array}\right\} \sum_{\rho, \rho_{c}=1,2}\left\langle(\lambda \mu) Y I ;(11) Y^{a} I^{a} \|\left(\lambda^{\prime} \mu^{\prime}\right) Y^{\prime} I^{\prime}\right\rangle_{\rho}
\end{aligned}
$$

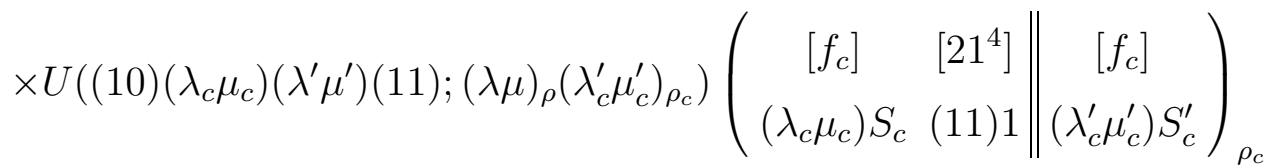

which contains a summation over the indices $\rho$ and $\rho_{c}$ related to the total system of $N_{c}$ quarks and to the core formed of $N_{c}-1$ quarks. One has $[f]=\left[N_{c}-2,1\right]$ for $p=1$ and $[f]=\left[N_{c}-1\right]$ for $p=2$. The $\mathrm{SU}(3)$ Racah coefficients $U$ appear due to the recoupling of the last quark. The matrix elements of the separated quark are simpler, as expected

$$
\left\langle\left[N_{c}-1,1\right] p ;\left(\lambda^{\prime} \mu^{\prime}\right) Y^{\prime} I^{\prime} I_{3}^{\prime} ; S^{\prime} m_{s}^{\prime}\left|g^{j a}\right|\left[N_{c}-1,1\right] p ;(\lambda \mu) Y I I_{3} ; S m_{s}\right\rangle=
$$




$$
\begin{aligned}
& (-1)^{S^{\prime}-1 / 2} \sqrt{2(2 S+1)}\left(\begin{array}{cc|c}
S & 1 & S^{\prime} \\
m_{s} & j & m_{s}^{\prime}
\end{array}\right)\left(\begin{array}{cc|c}
I & I^{a} & I^{\prime} \\
I_{3} & I_{3}^{a} & I_{3}^{\prime}
\end{array}\right) \\
& \times \sum_{p^{\prime}, p^{\prime \prime}, q^{\prime}, q^{\prime \prime}}(-1)^{S_{c}} K\left(\left[f^{\prime}\right] p^{\prime}\left[f^{\prime \prime}\right] p^{\prime \prime} \mid\left[N_{c}-1,1\right] p\right) K\left(\left[f^{\prime}\right] q^{\prime}\left[f^{\prime \prime}\right] q^{\prime \prime} \mid\left[N_{c}-1,1\right] p\right)\left\{\begin{array}{ccc}
S & 1 & S^{\prime} \\
1 / 2 & S_{c} & 1 / 2
\end{array}\right\} \\
& \times \sum_{\rho=1,2}\left\langle(\lambda \mu) Y I ;(11) Y^{a} I^{a}||\left(\lambda^{\prime} \mu^{\prime}\right) Y^{\prime} I^{\prime}\right\rangle_{\rho} U\left(\left(\lambda_{c} \mu_{c}\right)(10)\left(\lambda^{\prime} \mu^{\prime}\right)(11) ;(\lambda \mu)(10)\right)_{\rho}
\end{aligned}
$$

They all contain the isoscalar factors $K$ given in the previous section. Inserting them in the above expressions, together with the isoscalar factors $\left\langle(\lambda \mu) Y I ;(11) Y^{a} I^{a} \|\left(\lambda^{\prime} \mu^{\prime}\right) Y^{\prime} I^{\prime}\right\rangle_{\rho}$ and the SU(3) Racah coefficients $U$ and performing the sums in (22) and (23) we can obtain the matrix elements described in the next section.

As just mentioned above, for the calculations of the $G_{c}^{i a}$ and $g^{i a}$ matrix elements one needs to derive some SU(3) Racah coefficients. For that purpose, we follow the method described by Hecht [24]. We have obtained the following formulas to be used in Eqs. (22) and (23)

$$
\begin{aligned}
& \left\langle\left(\lambda_{c}^{\prime} \mu_{c}^{\prime}\right) Y_{c}^{\prime} I_{c}^{\prime} ;(10) y i \|\left(\lambda^{\prime} \mu^{\prime}\right) Y^{\prime} I^{\prime}\right\rangle U\left((10)\left(\lambda_{c} \mu_{c}\right)\left(\lambda^{\prime} \mu^{\prime}\right)(11) ;(\lambda \mu)_{\rho}\left(\lambda_{c}^{\prime} \mu_{c}^{\prime}\right)_{\rho_{c}}\right)= \\
& \sum_{\substack{Y_{c}, Y_{a}, Y, I_{c}, I_{a}, I}}(-1)^{\lambda_{c}-\lambda+\mu-\mu_{c}}(-1)^{\lambda_{c}^{\prime}-\lambda^{\prime}+\mu^{\prime}-\mu_{c}^{\prime}}(-1)^{i+I_{a}+I+I_{c}^{\prime}} \sqrt{(2 I+1)\left(2 I_{c}^{\prime}+1\right)}\left\{\begin{array}{ccc}
i & I_{c} & I \\
I_{a} & I^{\prime} & I_{c}^{\prime}
\end{array}\right\} \\
& \times\left\langle\left(\lambda_{c} \mu_{c}\right) Y_{c} I_{c} ;(11) Y_{a} I_{a} \|\left(\lambda_{c}^{\prime} \mu_{c}^{\prime}\right) Y_{c}^{\prime} I_{c}^{\prime}\right\rangle_{\rho_{c}}\left\langle(\lambda \mu) Y I ;(11) Y_{a} I_{a} \|\left(\lambda^{\prime} \mu^{\prime}\right) Y^{\prime} I^{\prime}\right\rangle_{\rho} \\
& \times\left\langle\left(\lambda_{c} \mu_{c}\right) Y_{c} I_{c} ;(10) y i \|(\lambda \mu) Y I\right\rangle \text {, }
\end{aligned}
$$

and

$$
\begin{aligned}
& \left\langle\left(\lambda_{c} \mu_{c}\right) Y_{c} I_{c} ;(10) y^{\prime} i^{\prime} \|\left(\lambda^{\prime} \mu^{\prime}\right) Y^{\prime} I^{\prime}\right\rangle U\left(\left(\lambda_{c} \mu_{c}\right)(10)\left(\lambda^{\prime} \mu^{\prime}\right)(11) ;(\lambda \mu)_{\rho}(10)\right)= \\
& \quad \sum_{\substack{y, Y_{a}, Y, i, I_{a}, I}}(-1)^{I_{c}+i+I^{\prime}+I_{a}} \sqrt{(2 I+1)\left(2 i^{\prime}+1\right)}\left\{\begin{array}{ccc}
I_{c} & i & I \\
I_{a} & I^{\prime} & i^{\prime}
\end{array}\right\}\left\langle\left(\lambda_{c} \mu_{c}\right) Y_{c} I_{c} ;(10) y i \|(\lambda \mu) Y I\right\rangle \\
& \quad \times\left\langle(10) y i ;(11) Y_{a} I_{a} \|(10) y^{\prime} i^{\prime}\right\rangle\left\langle(\lambda \mu) Y I ;(11) Y_{a} I_{a} \|\left(\lambda^{\prime} \mu^{\prime}\right) Y^{\prime} I^{\prime}\right\rangle_{\rho} .
\end{aligned}
$$

The required isoscalar factors can be found in Refs. [24, 25].

\section{B. Results}

Our analytic results for the non-vanishing isoscalar factors

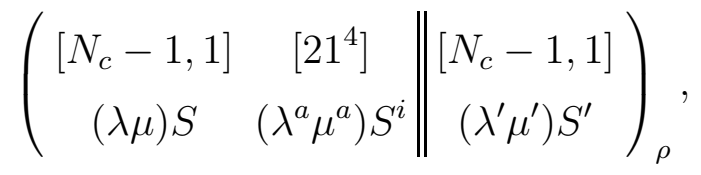


associated to the matrix elements of $G^{i a}$ as defined in Eq. (13) together with the normalization (2) are exhibited in Tables [I, II, III and IV] For $N_{c}=3$ they correspond to ${ }^{2} 8,{ }^{4} 8$,

${ }^{2} 10$ and ${ }^{2} 1$ multiplets respectively. To make the applications easier they are expressed in terms of $N_{c}$ and the spin of the total system which is fixed for each multiplet, namely $1 / 2$, $3 / 2,1 / 2$ and $1 / 2$ respectively. The values of the spin are consistent with the label of the corresponding $\mathrm{SU}(3)$ irreducible representation $(\lambda \mu)$ as illustrated by the examples (8)-(11), i.e. one has $\lambda=2 S$ and $\mu=\left(N_{c}-2 S\right) / 2$ for all the multiplets. The index $\rho$ is specified whenever necessary, with its two distinct values 1 and 2 .

\section{THE MASS OPERATOR OF STRANGE AND NON-STRANGE BARYONS}

It is very important to apply the $1 / N_{c}$ expansion method to both non-strange and strange baryons together. First, we have at our disposal a larger number of experimental data than for non-strange baryons alone and second, we can get a unified picture of all light baryons.

When the $\mathrm{SU}(3)$-flavor symmetry is exact, the $1 / N_{c}$ expansion mass operator describing an excited state can be written as the linear combination

$$
M^{(1)}=\sum_{i} c_{i} O_{i},
$$

where $c_{i}$ are unknown coefficients which parametrize the QCD dynamics and the operators $O_{i}$ are of type

$$
O_{i}=\frac{1}{N_{c}^{n-1}} O_{\ell}^{(k)} \cdot O_{S F}^{(k)}
$$

where $O_{\ell}^{(k)}$ is a $k$-rank tensor in $\mathrm{SO}(3)$ and $O_{S F}^{(k)}$ a $k$-rank tensor in $\mathrm{SU}(2)$, but scalar in $\mathrm{SU}(3)$-flavor (as shown by the upper index of $M^{(1)}$ ). This implies that $O_{i}$ is a combination of $\mathrm{SO}(3)$ generators $L^{i}$ and of $\mathrm{SU}(6)$ generators. The presence of $L^{i}$ is necessary in describing excited states.

When the $\mathrm{SU}(3)$-flavor symmetry is broken the mass operator in the $1 / N_{c}$ expansion contains additional terms, as first performed in Ref. [26] for the symmetric baryon multiplet

$$
M=\sum_{i} c_{i} O_{i}+\sum_{i} d_{i} B_{i}
$$

where the operators $B_{i}$ are defined to have zero expectation values for nonstrange quarks. The values of the coefficients $c_{i}$ and $d_{i}$ are found by a numerical fit to data. 


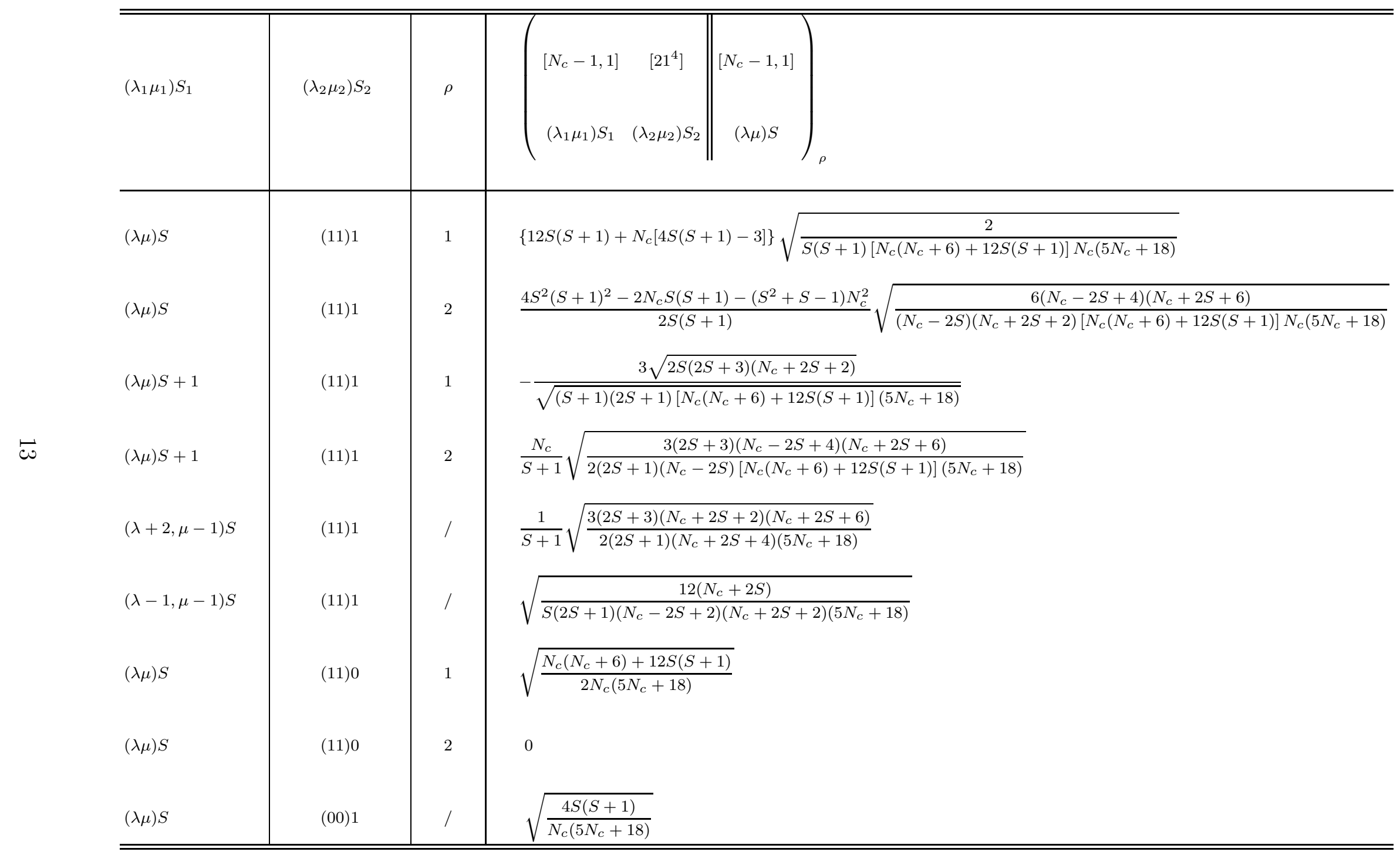

TABLE I: Isoscalar factors of the SU(6) generators, Eqs. (22) and (13), corresponding to the ${ }^{2} 8$ multiplet. 


\begin{tabular}{|c|c|c|c|}
\hline$\left(\lambda_{1} \mu_{1}\right) S_{1}$ & $\left(\lambda_{2} \mu_{2}\right) S_{2}$ & $\rho$ & $\left(\begin{array}{cc}{\left[N_{c}-1,1\right]} & {\left[21^{4}\right]} \\
\left(\lambda_{1} \mu_{1}\right) S_{1} & \left(\lambda_{2} \mu_{2}\right) S_{2}\end{array} \| \begin{array}{c}{\left[N_{c}-1,1\right]} \\
(\lambda-2, \mu+1) S\end{array}\right)_{\rho}$ \\
\hline$(\lambda-2, \mu+1) S$ & $(11) 1$ & 1 & {$\left[N_{c}(4 S-3)+6 S\right] \sqrt{\frac{2(S+1)}{S\left[N_{c}\left(N_{c}+6\right)+12(S-1) S\right] N_{c}\left(5 N_{c}+18\right)}}$} \\
\hline$(\lambda-2, \mu+1) S$ & $(11) 1$ & 2 & $-\frac{N_{c}-2 S}{S} \sqrt{\frac{3(S-1)(S+1)\left(N_{c}-2 S+6\right)\left(N_{c}+2 S\right)\left(N_{c}+2 S+4\right)}{2\left(N_{c}-2 S+2\right)\left[N_{c}\left(N_{c}+6\right)+12(S-1) S\right] N_{c}\left(5 N_{c}+18\right)}}$ \\
\hline$(\lambda \mu) S-1$ & $(11) 1$ & / & $\frac{N_{c}+4 S^{2}}{S} \sqrt{\frac{3\left(N_{c}+2 S+4\right)}{2(2 S-1)(2 S+1)\left(N_{c}+2 S+2\right) N_{c}\left(5 N_{c}+18\right)}}$ \\
\hline$(\lambda-2, \mu+1) S-1$ & (11)1 & 1 & $\frac{3 \sqrt{2(S-1)\left(N_{c}+2 S\right)}}{\sqrt{S\left[N_{c}\left(N_{c}+6\right)+12(S-1) S\right]\left(5 N_{c}+18\right)}}$ \\
\hline$(\lambda-2, \mu+1) S-1$ & $(11) 1$ & 2 & $-\frac{N_{c}}{S} \sqrt{\frac{3\left(N_{c}-2 S+6\right)\left(N_{c}+2 S+4\right)}{2\left(N_{c}-2 S+2\right)\left[N_{c}\left(N_{c}+6\right)+12(S-1) S\right]\left(5 N_{c}+18\right)}}$ \\
\hline$(\lambda-3, \mu) S-1$ & $(11) 1$ & / & $-2 \sqrt{\frac{3(S-1)\left(N_{c}+2 S-2\right)}{(2 S-1)\left(N_{c}-2 S+4\right) N_{c}\left(5 N_{c}+18\right)}}$ \\
\hline$(\lambda-2, \mu+1) S$ & $(11) 0$ & 1 & $\sqrt{\frac{N_{c}\left(N_{c}+6\right)+12(S-1) S}{2 N_{c}\left(5 N_{c}+18\right)}}$ \\
\hline$(\lambda-2, \mu+1) S$ & $(11) 0$ & 2 & 0 \\
\hline$(\lambda-2, \mu+1) S$ & $(00) 1$ & / & $\sqrt{\frac{4 S(S+1)}{N_{c}\left(5 N_{c}+18\right)}}$ \\
\hline
\end{tabular}

TABLE II: Isoscalar factors of the SU(6) generators, Eqs. (22) and (13), corresponding to the ${ }^{4} 8$ multiplet. 


\begin{tabular}{|c|c|c|c|}
\hline$\left(\lambda_{1} \mu_{1}\right) S_{1}$ & $\left(\lambda_{2} \mu_{2}\right) S_{2}$ & $\rho$ & $\left.\left(\begin{array}{cc||c|c}{\left[N_{c}-1,1\right]} & {\left[21^{4}\right]} & {\left[N_{c}-1,1\right]} \\
\left(\lambda_{1} \mu_{1}\right) S_{1} & \left(\lambda_{2} \mu_{2}\right) S_{2}\end{array}\right)_{\rho}(\lambda+2, \mu-1) S\right)_{\rho}$ \\
\hline$(\lambda+2, \mu-1) S$ & (11)1 & 1 & {$\left[N_{c}(4 S+7)+6(S+1)\right] \sqrt{\frac{2 S}{(S+1)\left[N_{c}\left(N_{c}+6\right)+12(S+1)(S+2)\right] N_{c}\left(5 N_{c}+18\right)}}$} \\
\hline$(\lambda+2, \mu-1) S$ & (11)1 & 2 & $-\frac{N_{c}+2(S+1)}{S+1} \sqrt{\frac{3 S(S+2)\left(N_{c}-2 S-2\right)\left(N_{c}-2 S+2\right)\left(N_{c}+2 S+8\right)}{2\left(N_{c}+2 S+4\right)\left[N_{c}\left(N_{c}+6\right)+12(S+1)(S+2)\right] N_{c}\left(5 N_{c}+18\right)}}$ \\
\hline$(\lambda \mu) S+1$ & (11)1 & I & $\frac{N_{c}+4(S+1)^{2}}{S+1} \sqrt{\frac{3\left(N_{c}-2 S+2\right)}{2(2 S+1)(2 S+3)\left(N_{c}-2 S\right) N_{c}\left(5 N_{c}+18\right)}}$ \\
\hline$(\lambda \mu) S$ & (11)1 & / & $-\frac{1}{S+1} \sqrt{\frac{3\left(N_{c}+2 S+2\right)\left(N_{c}-2 S+2\right)}{2\left(N_{c}-2 S\right)\left(5 N_{c}+18\right)}}$ \\
\hline$(\lambda+2, \mu-1) S$ & $(11) 0$ & 1 & $\sqrt{\frac{N_{c}\left(N_{c}+6\right)+12(S+1)(S+2)}{2 N_{c}\left(5 N_{c}+18\right)}}$ \\
\hline$(\lambda+2, \mu-1) S$ & (11)0 & 2 & 0 \\
\hline$(\lambda+2, \mu-1) S$ & $(00) 1$ & l & $\sqrt{\frac{4 S(S+1)}{N_{c}\left(5 N_{c}+18\right)}}$ \\
\hline
\end{tabular}

TABLE III: Isoscalar factors of the SU(6) generators, Eqs. (21) and (13), corresponding to the ${ }^{2} 10$ multiplet. 


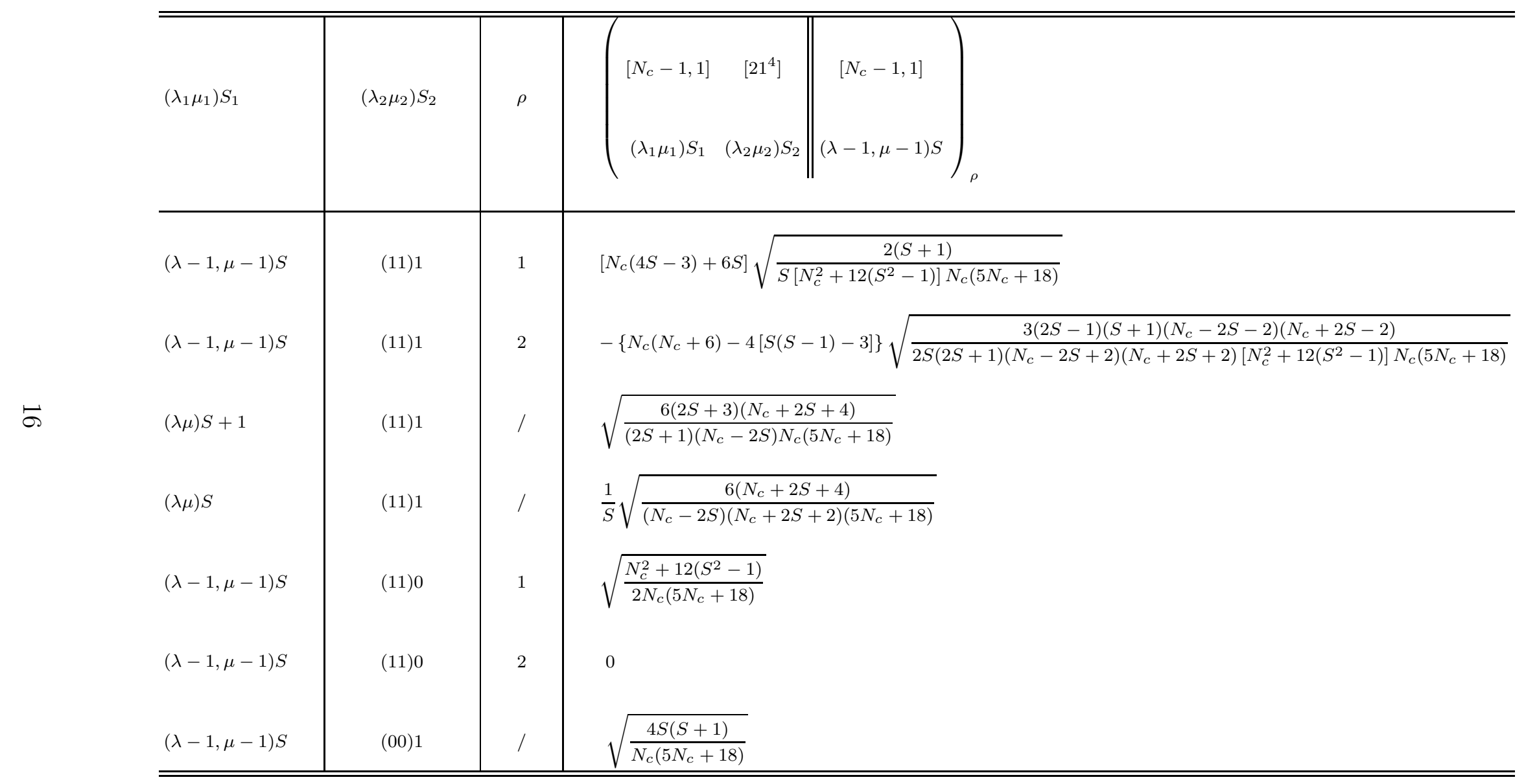

TABLE IV: Isoscalar factors of the SU(6) generators, Eqs. (21) and (13), corresponding to the ${ }^{2} 1$ multiplet. 
An essential step is to find all linearly independent operators contributing to a given order $\mathcal{O}\left(1 / N_{c}\right)$. In Table $\mathrm{V}$ we present a list of operators expected to be dominant up to order $1 / N_{c}$. The order of their matrix elements in $\mathrm{SU}(6)$, indicated in the second column of the table is not always the same as in $\mathrm{SU}(4)$ [10]. For example the operator $O_{4}$ is of order $N_{c}^{0}$ while in $\mathrm{SU}(4)$ is of order $1 / N_{c}$. This can be understood by looking at its matrix elements obtained from the relations (15)-(17)

$$
\frac{1}{N_{c}} T^{a} T^{a}=\frac{1}{12 N_{c}}\left\{N_{c}\left(N_{c}+6\right)+3 \lambda(\lambda+2)-3 f\left[2\left(N_{c}+3\right)-3 f\right]\right\},
$$

with $\lambda, \mu$ and $f$ illustrated by the example in Figure1, Let us remind that for any irreducible representation of SU(3) two labels are enough. Usually one takes $\lambda$ and $\mu$. For the present discussion, where $N_{c}$ is needed, it is more convenient to use $\lambda$ and $f$. For a system of $N_{c}$ quarks one has $\mu=\left(N_{c}-\lambda-3 f\right) / 2$, which leads to Eq. (30). When $N_{c}=3$ one has $f=0$ for the octet and the decuplet and $f=1$ for the singlet of $\mathrm{SU}(3)$. By looking at Eq. (30), one can notice that the first term in the bracket, $N_{c}\left(N_{c}+6\right)$, which is responsible for the order of the operator $\mathrm{O}_{4}$, is the same for all representations. That justifies the new definition introduced in Table $\mathrm{V}$ where $\left(N_{c}+6\right) / 12$ has been subtracted. It is important to stress that this new definition of $\mathrm{O}_{4}$ gives the same matrix elements as the isospin-isospin operator $1 / N_{c}\left(T^{a} T^{a}\right)$, used in Ref. [10] for non-strange baryons ( $\lambda=2 I$ for non-strange baryons). The new operator $\mathrm{O}_{4}$ is then a natural generalization to $\mathrm{SU}(3)$ of its $\mathrm{SU}(2)$-isospin counterpart.

An important property is that the order of $O_{4}$ is now $N_{c}^{0}$ and not $1 / N_{c}$ as one would expect from $\mathrm{SU}(4)$. This comes from the third term of Eq. (30) which contributes only for SU(6) representations which become singlets for $N_{c}=3$, as explained above. The $\mathrm{SU}(6)$ symmetry is then broken to order 1 in the large $N_{c}$ limit for the mixed symmetric representation $\left[N_{c}-1,1\right]$. This result, which does not appear for ground state baryons, should be analyzed in more details in the future. Meanwhile one can notice that the order $N_{c}^{0}$ of $O_{4}$ is consistent with Eqs. (18) and (19) of Ref. [27] where one predicts five towers of states and where the singlet always belongs to different towers from the octet and the decuplet. By adding more operators in the restricted list of Table $\square$ as, for example, $L^{(2) i j} G^{i a} G^{j a}$, we might expect to obtain results consistent with Ref. [27] in physical applications.

The order of $O_{5}$ and $O_{6}$ follow from the arguments given in Ref. [28]. Accordingly, unlike the case of two light flavors, the matrix elements of the flavor generators $T^{a}$ and spin-flavor generators $G^{i a}$ do not have the same $N_{c}$ dependence everywhere in the flavor weight diagram. 


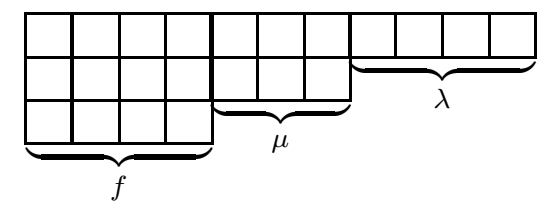

FIG. 1: Young diagram of an SU(3) irreducible representation.

\begin{tabular}{lr}
\hline \hline Operator & Matrix elements order in $\mathrm{SU}(6)$ \\
\hline$O_{1}=N_{c} \mathbb{1}$ & $N_{c}$ \\
$O_{2}=L^{i} S^{i}$ & $N_{c}^{0}$ \\
$O_{3}=\frac{1}{N_{c}} S^{i} S^{i}$ & $N_{c}^{-1}$ \\
$O_{4}=\frac{1}{N_{c}}\left(T^{a} T^{a}-\frac{1}{12} N_{c}\left(N_{c}+6\right)\right)$ & $N_{c}^{0}$ \\
$O_{5}=\frac{3}{N_{c}} L^{i} T^{a} G^{i a}$ & $N_{c}^{0}$ \\
$O_{6}=\frac{3}{N_{c}^{2}} S^{i} T^{a} G^{i a}$ & $N_{c}^{-1}$ \\
$O_{7}=\frac{1}{N_{c}} L^{(2) i j} S^{i} S^{i}$ & $N_{c}^{-1}$ \\
\hline$B_{1}=\mathcal{S}$ & $N_{c}^{0}$ \\
\hline \hline
\end{tabular}

TABLE V: Examples of operators $O_{i}$ and $B_{1}$ entering the mass formula.

The baryons under concern, located at the top of an $\mathrm{SU}(3)$ weight diagram, therefore having finite strangeness, have matrix elements of $T^{a}$ which are of order $\mathcal{O}(1), \mathcal{O}\left(\sqrt{N_{c}}\right)$ and $\mathcal{O}\left(N_{c}\right)$ for $a=1,2,3, a=4,5,6,7$ and $a=8$ respectively and matrix elements of $G^{i a}$ which are $\mathcal{O}\left(N_{c}\right), \mathcal{O}\left(\sqrt{N_{c}}\right)$ and $\mathcal{O}(1)$. Then the corresponding combinations give for $O_{5}$ and $O_{6}$ the order $N_{c}^{0}$ and $N_{c}^{-1}$ respectively. $B_{1}$ is of course of order $N_{c}^{0}$.

The contribution of $\mathrm{SO}(3)$, is first manifested in the operator $\mathrm{O}_{2}$ which represents the spin-orbit coupling where $L^{i}$ and $S^{i}$ are the total angular momentum and spin components. In applications one can also use the Hartree approximation [5] which is a one-body operator. This approximation is useful because it shows that the leading order of the spin-orbit contribution is $N_{c}^{0}$. Lastly, the operator $O_{7}$ contains the $\mathrm{SO}(3) 2 \mathrm{nd}$ rank tensor, defined as

$$
L^{(2) i j}=\frac{1}{2}\left\{L^{i}, L^{j}\right\}-\frac{1}{3} \delta_{i,-j} \vec{L} \cdot \vec{L},
$$


which, like $L^{i}$, acts on the orbital wave function $\left|L m_{L}\right\rangle$ of the whole system of $N_{c}$ quarks.

In $\mathrm{SU}(4)$ the practice on the $\left[\mathbf{7 0}, 1^{-}\right]$multiplet [10] showed that the operators $O_{1}, O_{3}$, $O_{4}$ (defined as $\frac{1}{N_{c}} T^{a} T^{a}$ ) and $O_{6}$ are the most dominant. A problem is to find out if this behavior also holds in $\mathrm{SU}(6)$. Also one has to reanalyze the $\left[\mathbf{7 0}, \ell^{+}\right]$multiplet, studied so far in the decoupling scheme only [8]. Applications to baryons belonging to the $\left[\mathbf{7 0}, \ell^{ \pm}\right]$ multiplets will be considered in subsequent studies.

We should finally mention, that the method based on the separation of the system into a symmetric core of $N_{c}-1$ quarks and an excited quark, as first used in Ref. [5], acquired some support from the work of Pirjol and Schat [29], based on a large $N_{c}$ quark model Hamiltonian, where explicit results for the coefficients $c_{i}$ are presented both for the one gluon exchange (OGE) and for the Goldstone boson exchange (GBE) hyperfine interactions, with radial dependent form factors. An extension of the study of Collins and Georgi [30] from $N_{c}=3$ to large $N_{c}$ is obtained in this way.

Including the space degree of freedom, Pirjol and Schat decompose the two-body operators into tensor operators transforming as $\mathrm{S}, \mathrm{MS}$ and E representations of $S_{N_{c}}$ (the latter exists only for $N_{c}>3$ ). Moreover, the splitting of the $\mathrm{SU}(4)$ generators into two pieces (see introduction), $S^{i}=S_{c}^{i}+s^{i}, T^{a}=T_{c}^{a}+t^{a}$ and $G^{i a}=G_{c}^{i a}+g^{i a}$, matches the choice of their basis states. The conclusion of Ref. [29] was that the inclusion of core and excited quark operators is necessary, at variance with our simplified procedure.

A useful result is that Pirjol and Schat obtain a large $N_{c}$ dependence similar to that of the $1 / N_{c}$ expansion method. The tower structure, first observed in the $\mathrm{N}=1$ band in Ref. [31], is satisfied at leading order in $1 / N_{c}$. The resulting mass formula contains 6 independent non-vanishing coefficients at order $1 / N_{c}$ both for OGE and for GBE. They have to be found by fitting the 7 resonance masses available in the $\mathrm{N}=1$ band. Therefore some arbitrariness is imposed in combining OGE with GBE. In general, the arbitrariness could lead to anomalies, as shown in Ref. [9].

On the other hand in the method based on the quark model Hamiltonian, the operator $S^{i} T^{a} G^{i a}$ is absent, but present in our case, see Table $\mathrm{V}$. In Ref. [10] we have shown that such an operator brings a dominant contribution to most of the nucleon masses in the $\mathrm{N}=1$ band. According to Ref. [5] this absence is allowed in SU(4) but, as explicitly stated there, for more than two flavors a term like $S_{c}^{i} t^{a} G_{c}^{i a}$, should be included in the mass operator. Thus the extension to $\mathrm{SU}(6)$ of the work of Pirjol and Schat could, at least in this respect, raise 
problems.

The basic difference between our work and that of Pirjol and Schat is the radial dependence is integrated out in our case, consistent with the $1 / N_{c}$ expansion method. When there is no radial dependence, the spin-spin operator is symmetric. The quadrupole operator of the quark model makes its presence through its symmetric part, which is related to the 2nd-rank SO(3) operator (31), as in the work of Collins and Georgi [30], or of Carlson et al. [5] and as in subsequent studies. So far, in applications, the spin-orbit term was treated in the Hartree approximation. In this context, our operator basis is complete inasmuch as the orbital part is always symmetric, thus the flavour-spin part should be symmetric too, so we have only $\left\langle\mathrm{MS}\left|O^{S}\right| \mathrm{MS}\right\rangle$ matrix elements in the flavour-spin space.

The differences between the two methods should be confronted in future applications to nonstrange and strange baryons, from where one wishes to obtain meaningful physical information on the coefficients $c_{i}$.

\section{CONCLUSIONS}

The isoscalar factors of the SU(6) generators derived in this study opens new applications of the $1 / N_{c}$ expansion method to baryons spectroscopy. It allows to combine data on nonstrange and strange baryons together and to lead to a more precise determination of the coefficients $c_{i}$ and $d_{i}$ which encode the QCD dynamics. Finally, our results presented in Tables I-IV, can be used for other $N$-body problems governed by $\mathrm{SU}(6)$ symmetry and where the spin is known.

[1] G. 't Hooft, Nucl. Phys. 72, 461 (1974).

[2] E. Witten, Nucl. Phys. B160 57, (1979).

[3] J. L. Gervais and B. Sakita, Phys. Rev. Lett. 52, 87 (1984); Phys. Rev. D 30, 1795 (1984).

[4] R. Dashen and A. V. Manohar, Phys. Lett. B 315 (1993) 425; ibid B 315, 438 (1993).

[5] C. E. Carlson, C. D. Carone, J. L. Goity and R. F. Lebed, Phys. Lett. B 438, 327 (1998); Phys. Rev. D 59, 114008 (1999).

[6] N. Matagne and Fl. Stancu, Phys. Lett. B 631, 7 (2005). 
[7] C. L. Schat, J. L. Goity and N. N. Scoccola, Phys. Rev. Lett. 88, 102002 (2002); J. L. Goity, C. L. Schat and N. N. Scoccola, Phys. Rev. D 66, 114014 (2002).

[8] N. Matagne and F. Stancu, Phys. Rev. D 74, 034014 (2006).

[9] N. Matagne and F. Stancu, Phys. Rev. D 77, 054026 (2008).

[10] N. Matagne and F. Stancu, Nucl. Phys. A811, 291 (2008).

[11] K. T. Hecht and S. C. Pang, J. Math. Phys. 10, 1571 (1969).

[12] C. L. Cook and G. Murtanza, Il Nuovo Cimento, 39, 531 (1965).

[13] J. C. Carter, J. J. Coyne and S. Meshkov, Phys. Rev. Lett. 13, 523 (1965).

[14] L. Schülke, Z. Phys. 183, 427 (1965).

[15] J. C. Carter and J. J. Coyne J. Math. Phys. 10, 1204 (1969).

[16] N. Matagne and F. Stancu, Phys. Rev. D 73, 114025 (2006).

[17] J. J. De Swart, Rev. Mod. Phys. 35, 916 (1963).

[18] Fl. Stancu and S. Pepin, Few-Body Systems 26, 113 (2004).

[19] D. B. Lichtenberg, Unitary symmetries and elementary particles, Academic Press, New York and London (1970), Chapter 8.

[20] A. de-Shalit and I. Talmi, Nuclear Shell Theory, Academic Press, New York, N.Y. (1963).

[21] K. T. Hecht 'Collective models' in Selected topics in nuclear spectroscopy (ed. by B. J. Verhaar), North Holland, Amsterdam, (1964).

[22] F. Stancu, Phys. Rev. D 58, 111501 (1998).

[23] Fl. Stancu, Group Theory in Subnuclear Physics, Oxford University Press, Oxford (1996) Chapter 4.

[24] K. T. Hecht, Nucl. Phys. 62, 1 (1965).

[25] J. D. Vergados, Nucl. Phys. A111, 681 (1968).

[26] E. E. Jenkins and R. F. Lebed, Phys. Rev. D 52, 282 (1995).

[27] T. D. Cohen and R. F. Lebed, Phys. Rev. D 72, 056001 (2005).

[28] E. Jenkins, Ann. Rev. Nucl. Sci. 48, 81 (1998).

[29] D. Pirjol and C. Schat, Phys. Rev. D 78, 034026 (2008).

[30] H. Collins and H. Georgi, Phys. Rev. D 59, 094010 (1999).

[31] D. Pirjol and T. M. Yan, Phys. Rev. D 57, 5434 (1998). 